606 Christomanos: Zur quantitativen Trennung von Kalk und Magnesia

\begin{tabular}{l|c|c|c|c|c|c}
\hline & $\mathbf{1}$ & 2 & 3 & 4 & 5 & 6 \\
\hline $\begin{array}{c}\text { Vorhandener } \\
\text { Holzschliff- } \\
\text { Gehalt in } \% 0\end{array}$ & 60,2 & 23,0 & 38,7 & 56,0 & 33,2 & 33,2 \\
\hline $\begin{array}{c}\text { Gefundener } \\
\text { Holzschliff- } \\
\text { Gehalt in } \%\end{array}$ & 59,6 & 23,3 & 38,5 & 55,8 & 33,1 & 33,8
\end{tabular}

wird ersichtlich, dass die angeführte quantitative Untersuchungsmethode befriedigende Resultate liefert.

Die Untersuchungen werden fortgesetzt.

Wi n, Chemisches Laboratorium der Wiener Handels-Akademie.

\title{
Zur quantitativen Trennung von Kalk und Magnesia auf indirectem Wege.
}

Die Magnesite Griechenlands.

\section{Von \\ Dr. A. C. Christomanos.}

Die quantitative Trennung von Kalk und Magnesia gestaltet sich manchmal, besonders beim Vorwiegen der Magnesia, so misslich, dass man trotz aller Vorsichtsmaassregeln immer wieder die Niederschläge des oxalsauren Kalks und des phosphorsauren Ammonmagnesiums auflösen und wieder fällen muss, um aus jenem Nagnesia, aus diesem aber noch Kalk auszuscheiden, worauf schon R. Fresenius'), R. Bunsen ${ }^{2}$ ) und Al. Classen ${ }^{3}$ ) aufmerksam machten.

1) Anleitung zur quantitativen chemischen Analyse, Braunschweig 1875, Bd. I, S. 556 .

2) Anleitung zur Analyse der Aschen und Mineralwasser, Feidelberg 1874, S. $25-26$.

3) Ausgewählte Methoden der analytischen Chemie, Braunschweig 1901, Bd. I, S. 836 . 
Ohne jetzt auf die Einzelheiten eines verbesserten Verfahrens bei dieser Trennung eingehen zu wollen, durch welches auch unter den missgünstigsten Umständen die Trennung sicher auszuführen ist, möchte ich hier nur einige Versuche angeben, nach welchen sich in einigen Fällen genaue Ergebnisse schnell erzielen liessen. Die Analytiker vermeiden mit Recht wo möglich die Anwendung der indirecten Analyse, da bei den erforderlichen Bestimmungen die grösste Genauigkeit obwalten muss, damit nicht die kleinsten Fehler grosse Abweichungen der Resultate verursachen. Dennoch sind bei der grossen Einfachheit und Schnelligkeit, mit welcher sich diese Bestimmungen ausführen lassen, die Methoden der indirecten Analyse oft indicirt. So zum Beispiel bei der Analyse der viel Magnesiumsalze enthaltenden Mineralwasser oder des Magnesits und des Dolomits.

\section{A. Bei der Mineralwasser-Analyse.}

Hat man entweder aus dem bei $180^{\circ}$ bis zum constanten Gewicht erhitzten Verdampfungsrückstand oder, nach dem Ausziehen desselben durch Wasser, aus dem unlöslichen Theil oder endlich aus dessen gelöstem Theil, die Kohlensäure bestimmt, die Kieselsäure, das Eisenoxyd und die Thonerde abgeschieden, so bleiben im Filtrat nur mehr die Salze des Kalks, der Magnesia und der Alkalien. Man verdampft diese Filtrate in einem gewogenen Porzellanschälchen zur Trockne unter Zusatz von Sodalösung, verjagt die Ammoniaksalze durch schliessliches Glühen, nimmt wieder mit Sodalösung auf and zieht endlich mit heissem Wasser alles Lösliche aus, worauf in der Schale bloss die beiden Carbonate des Calciums und Magnesiums zurückblieben, die man auf $200^{\circ}$ bis zu constantem Gewicht erhitzt. Man erhält so das Gewicht a der beiden Carbonate.

Nun mengt man im Schälchen oder in einem etwas geräumigen Porzellantiegel die Carbonate mit einigen Tropfen Wasser und vorsichtig mit einer hinreichenden Menge concentrirter Schwefelsäure, erhitzt mit aufgelegtem Deckel erst auf dem Sandbade und dann mit kleiner freier Flamme bis zum Abrauchen der Schwefelsäure, wiederholt dies mit noch einigen Tropfen dieser Säure, vermischt mit etwas Ammoniumcarbonat (Pulver oder concentrirte Lösung) und glüht wieder bis zum 
608 Christomanos: Zur quantitativen Trennung von Kalk und Magnesia

constanten Gewicht, welches nun das gemeinschaftliche Gewicht b der Sulfate des Calciums und des Magnesiums darstellt.

B. Bei der Analyse von Magnesit.

Bei Gelegenheit dieser Publication sei es gestattet, eine Zusammenstellung der Analysen aller bekannten Magnesite Griechenlands wiederzugeben, um auf dieses wichtige and unübertrefflich reiche and reine Naturproduct die Aufmerksamkeit der Mineralogen und hauptsächlich der Industrie Deutschlands zu lenken. In Mantudi, auf der Nordostküste der Insel Euböa, hat die griechische Gesellschaft » Société des travaux publics et municipaux ein grosses Calcinir-Etablissement errichtet zur Verarbeitung des von ihr in Mantudi und Limni geförderten Magnesits, in welchem sie gebrannte Magnesia, die sie kaustische nennt, totgebrannte Magnesia zur Cementfabrikation und ganz besonders feuerfeste Magnesiaziegel mit und ohne Chromeisensteinzusatz durch Erhitzen in Mendheim'schen Oefen bis auf $2100^{\circ}$ darstellt, wozu sie, allerdings etwas verschwenderisch, die ebenfalls von ihr geförderten griechischen Lignite von Kumi auf Euböa verwendet. Auf der Westküste Euböa's, bei Limni, hat ferner die englische Gesellschaft Petrifite eine noch grössere Installation mit Eisenbahn gegründet, um Magnesit zu calciniren.

Der Gehalt der griechischen Magnesite an Magnesiumcarbonat, den man aus der folgenden Tabelle durch Berechnung des Magnesiagehaltes mit dem zur Bildung von neutralem Carbonat nöthigen Kohlendioxyd ersehen kann, ist erstaunlich und beträgt manchmal bis zu $98,75 \%$. Das Mineral ist von dichter Beschaffenheit und blendend weiss and sind besonders die scheiben- oder tellerförmigen Gebilde fast chemisch rein. Die Kieselsäure durchzieht sporadisch in Form glasglänzender Schnüre das mächtige Vorkommen; die Thonerde stammt aus zersetztem Serpentinfels der Solbänder. Eisenoxydul kommt auch nicht spurenweise vor und das Eisenoxyd häuft sich besonders, wenn auch nur in sehr kleinen Mengen, in Rissen und Spalten des Minerals an, die dann gelblich erscheinen.

Die griechischen Magnesite, die hier ausschliesslich zur Analyse kamen, haben folgende Zusammensetzung: 


\begin{tabular}{|c|c|}
\hline$\sum_{\substack{\infty \\
\sin _{0}}}^{\infty} \frac{0}{0}$ & 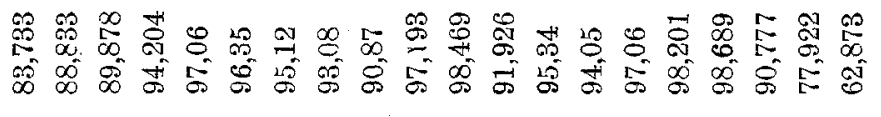 \\
\hline 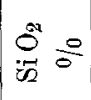 & 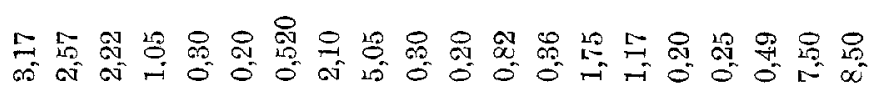 \\
\hline 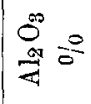 & 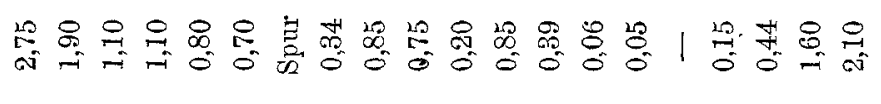 \\
\hline$\underset{\substack{0 \\
\mathbb{N}_{1}}}{\infty}$ & 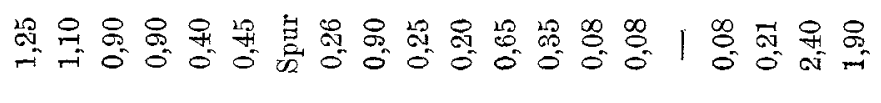 \\
\hline$\stackrel{0}{10}$ & 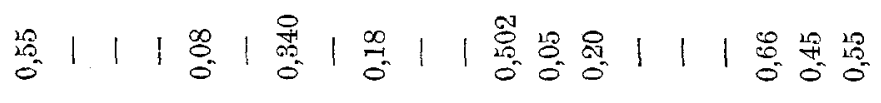 \\
\hline 80 & 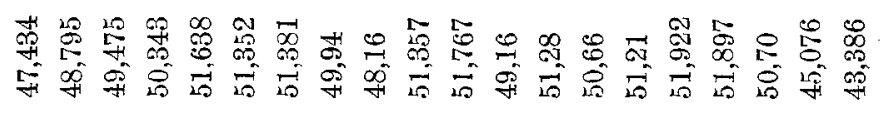 \\
\hline 迸 & 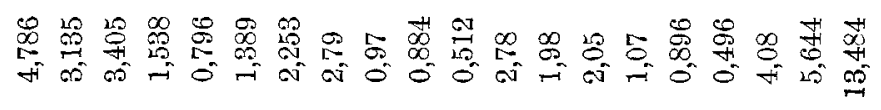 \\
\hline $\int_{i=0}^{0}$ & 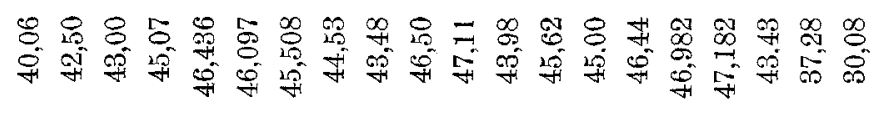 \\
\hline 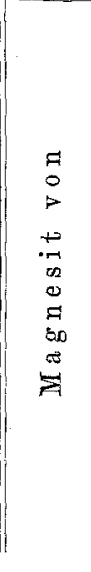 & 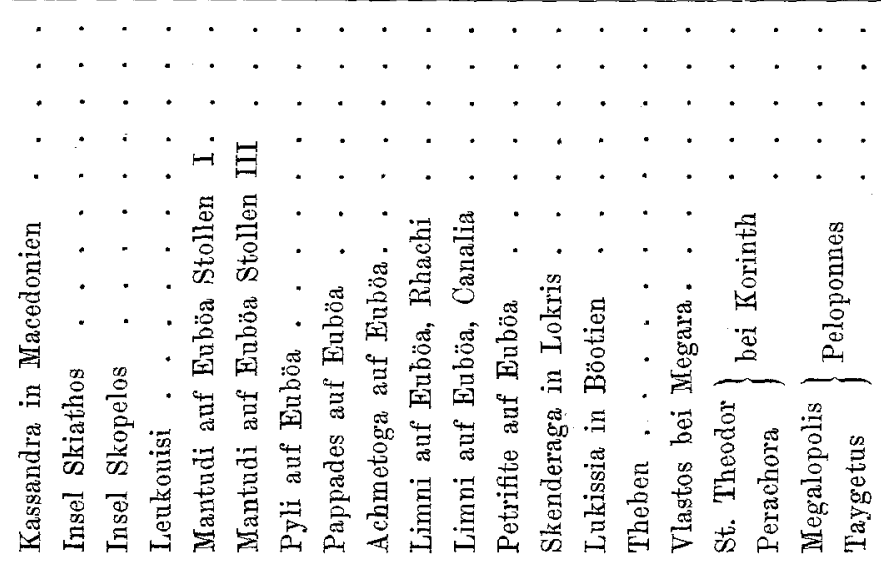 \\
\hline
\end{tabular}


610 Christomanos: Zur quantitativen Trennung von Kalk und Magnesia

Hat man in einer eigenen Probe die Kieselsäure, das Eisenoxyd, die Thonerde und das Wasser bestimmt, so kennt man auch schon das Gewicht a der Carbonate. Durch halbstündiges Glühen des scharf getrockneten Mineralpulvers im Platintiegel vor dem Gebläse erhält man das Gewicht der Oxyde des Calciums und des Magnesiums und der entwichenen Kohlensäure und durch Behandlung dieses Rückstandes oder des ursprünglichen, scharf getrockneten Mineralpulvers in demselben Tiegel mit Schwefelsäure, nach Abzug der Kieselsäure, des Eisenoxyds und der nur unbedeutend veränderten Thonerde, das Gewicht $b$ der entsprecenden Sulfate.

1. Indirecte $\mathrm{Bestimmung}$ von $\mathrm{CaO}$ und $\mathrm{MgO}$ aus dem Gewichte der Carbonate und der Sulfate.

Unter der Voraussetzung, dass die beiden Carbonate und Sulfate in Quantitäten gleicher Molecüle vorhanden wären, hätten wir

$$
\begin{aligned}
\mathrm{CaCO} \mathrm{CO}_{3}+\mathrm{MgCO}_{3}=a=184,36 \\
\text { und } \mathrm{Ca} \mathrm{SO} \mathrm{SO}_{4}+\mathrm{Mg} \mathrm{SO}_{4}=\mathrm{b}=256,48
\end{aligned}
$$

$\mathrm{Da}$ in beiden dieselbe Menge $\mathrm{CaO}$ und $\mathrm{MgO}$ rorhanden ist, nennen wir diese Menge

$$
\begin{aligned}
\mathrm{CaO} & =\mathrm{x}=56 \\
\operatorname{Mg} \mathrm{O} & =\mathrm{z}=40,36
\end{aligned}
$$

Bezeichnen nun die Zahlen $\mathrm{x}$ und $\mathrm{z}$ nicht die Moleculargewichte, sondern beliebige Mengen von $\mathrm{CaO}$ und $\mathrm{Mg} \mathrm{O}$, so werden natürlich auch die Werthe von $a$ und $b$ verändert werden, aber sie werden stets im obigen Sinne von den Werthen $x$ und $z$ abhängig sein, und wir erhalten allgemein die Gleichungen:

$$
\begin{aligned}
& \text { 1. } f x+h z=a, \\
& \text { 2. } f_{1} x+h_{1} z=b .
\end{aligned}
$$

Unter der obigen Voraussetzung müssen diese Coëfficienten, mit $\mathrm{x}$ und $\mathrm{z}$ multiplicirt, die angenommenen Molecularmengen von $\mathrm{Ca} \mathrm{CO}_{3}$, $\mathrm{Mg} \mathrm{CO}, \mathrm{Ca} \mathrm{SO}_{4}$ und $\mathrm{MgSO}_{4}$ ergeben:

$f$ mal $\times$ gibt $\mathrm{CaCO}$; in unserem Falle $=100$ also $f=1,7857142857$

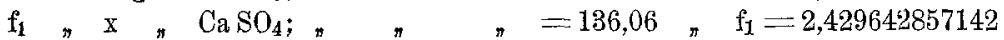

$\mathrm{h}$, $\mathrm{z}, \mathrm{MgCO}_{3} ;$, $,=84,36, \mathrm{~h}=2,0901883052$

$\mathrm{h}_{1}$ " $\mathrm{z}$ " $\mathrm{MgSO} \mathrm{SO}_{4} ; \%, \quad "=120,42, \mathrm{~h}_{1}=2,9836471754$

Aus Gleichung 1) ist $x=\frac{a-h z}{f}$, welcher Werth, in die Gleichung 2) substituirt, gibt

$$
\mathrm{f}_{1}\left(\frac{\mathrm{a}-\mathrm{hz}}{\mathrm{f}}\right)+\mathrm{h}_{1} \mathrm{z}=\mathrm{b}
$$


woraus

$$
\mathrm{z}=\frac{\mathrm{fb}-\mathrm{f}_{\mathrm{1}} \mathrm{a}}{\mathrm{fh}_{1}-\mathrm{f}_{1} \mathrm{~h}}
$$

dieser Werth von $z$ in Gleichung 1 ) gebracht gibt

und daraus

$$
f x+h\left(\frac{f b-f_{1} a}{f h_{1}-f_{1} h}\right)=a
$$

$$
\mathrm{x}=\frac{\mathrm{h}_{1} \mathrm{a}-\mathrm{h} b}{\mathrm{fh}_{1}-\mathrm{f}_{1} \mathrm{~h}} \text {. }
$$

Setzt man nun die Werthe der Coëficienten ein, so ergibt sich:

$$
\begin{aligned}
& x=11,957135 a-8,376556 b \\
& z=7,15635 \quad b-9,73693 a
\end{aligned}
$$

2. Indirecte Bestimmung aus dem Gewichte der Carbonate und den daraus durch Glüben gebildeten Oxyden:

$$
\begin{aligned}
& \text { 1) } \mathrm{Ca} \mathrm{CO}_{3}+\mathrm{MgCO}=\mathrm{a} \text { folglich } \mathrm{fx}+\mathrm{hz}=\mathrm{a} \\
& \text { 2) } \mathrm{CaO}+\mathrm{MgO}=\mathrm{c} \quad \mathrm{x}+\mathrm{z}=\mathrm{c} \\
& \mathrm{x}=\frac{\mathrm{hb}-\mathrm{a}}{\mathrm{h}-\mathrm{f}}=6,864912275 \mathrm{~b}-3,28434937 \mathrm{a} \\
& \mathrm{z}=\frac{\mathrm{a}-\mathrm{fb}}{\mathrm{h}-\mathrm{f}}=3,28434937 \mathrm{a}-5,864912275 \mathrm{~b}
\end{aligned}
$$

3. Indirecte Bestimmung des Calciumcarbonats und des Magnesiumcarbonats des Magnesits oder eines Gemenges derselben durch die blosse Bestimmung des Kohlendioxyds oder auch des Glühverlustes.

Man wägt die gepulverten Carbonate (a) im Platintiegel and hestimmt deren Glühverlust, darauf bringt man sie in den kleinen Kohlensäurebestimmungsapparat ${ }^{1}$ ), der absolut genaue Zahlen gibt, und bestimmt so das Kohlendioxyd (d). Bei der alleinigen Bestimmung des Kohlendioxyds aus dem Glühverlust erhält man bloss approximative Resultate, da dasselbe nur äusserst schwer gänzlich aus dem Magnesiumcarbonat auszutreiben ist.

$$
\begin{aligned}
& \mathrm{CaCO}^{3}+\mathrm{MgCO}_{3}=\mathrm{a} . \\
& \text { Nennt man } \mathrm{CaCO}_{3}=\mathrm{x}^{1} \\
& \qquad \mathrm{MgO}_{3}=\mathrm{z}^{1}, \text { so ergibt sich } \\
& \text { 1) } \mathrm{x}^{1}+\mathrm{z}^{1}=\mathrm{a} \\
& \text { 2) } \mathrm{mx}_{1}+\mathrm{nz}_{1}=\mathrm{d}
\end{aligned}
$$

wobei $m$ und $n$ Coëfficienten sind, die mit $x_{1}\left(\mathrm{CaCO}_{3}=100\right)$ und $z_{1}$ $\left(\mathrm{Mg} \mathrm{CO} \mathrm{C}_{3}=84,36\right)$ multiplirt $\mathrm{CO}_{2}=44$ geben.

1) Ber. d. deutsch. chem. Gesellsch. zu Berlin 27, 2748 (1894). 


$$
\begin{aligned}
\mathrm{m} & =0,44 \\
\mathrm{n} & =0,52157421 \\
\mathrm{x}_{1} & =6,3938616 \mathrm{a}-12,2587764 \mathrm{~d} \\
\mathrm{z}_{1} & =12,2587764 \mathrm{~d}-5,3938616 \mathrm{a} .
\end{aligned}
$$

Wie man sieht erheischen diese Methoden die einfachsten Manipulationen und beanspruchen fast keinen Zeitaufwand; nur müssen die erforderlichen Bestimmungen mit der möglichsten Genauigkeit ausgeführt werden, denn sonst gibt es ungeheure Abweichungen, eventuell sogar negative Zahlen, wie überhaupt bei den meisten indirecten Analysen.

Universitätslaboratorium Athen.

\section{Ueber die Bestimmung des Nitratstickstoffes neben organischem Stickstoff. Von}

\section{Th. Pfeiffer.}

Vor einigen Jahren liabe ich in Gemeinschaft mit meinem damaligen Mitarbeiter H. Thurmann über Versuche berichtet ${ }^{1}$ ), die wir zur Gewinnung einer brauchbaren Methode für die Bestimmung des Nitratstickstoffs neben organischem Stickstoff angestellt hatten und wobei wir unter Anderem zu dem Ergebnisse gelangt waren, dass das bekannte Schlösing'sche Verfahren, bei Gegenwart von leicht zersetzlichen Amiden, beziehungsweise auch von Ammoniumverbindungen, zu niedrige Zahlen liefert.

Im Gegensatz hierzu vertreten P. Liechti und E. Ritter in einer kürzlich erschienenen Arbeit ${ }^{2}$ ) unter Zurückweisung unserer Angaben den Standpunkt, dass die Schlösing'sche Methode für den Zweck, für den sie geschaffen wurde, nämlich die Bestimmung der Salpetersäure bei Gegenwart organischer Substanzen, in vorzüglicher Weise brauchbar und, wie schon ihr Schöpfer sagt, genau und allgemeiner Anwendung fähig sei.

Ich musste infolge dessen selbstverständlich die früheren Untersuchungen einer erneuten Prüfung unterwerfen and bin hierbei in Gemeinschaft mit meinem jetzigen Mitarbeiter R. Riecke zu folgenden Ergebnissen gelangt.

1) Landwirthsch. Versuchsstationen 46, 1 (1896).

2) Diese Zeitschrift 42, 205. 